\title{
Über die Verdauung einiger Eiweißkörper durch Pankreasfermente.
}

\author{
Von
}

\author{
Emil Fischer und Emil Abderhalden.
}

(Aus dem I. chemischen Institut der Universität Berlin.)

(Der Redaktion zugegangen am 11. Juni 1903.)

Bei der Hydrolyse der meisten Proteinstoffe durch kochende Säuren entsteht, wie durch eine Reihe von Arbeiten aus dem hiesigen Institute nachgewiesen wurde, eine nicht unbeträchtliche Menge von $\alpha$-Pyrrolidincarbonsäure. Für dàs Casein speziell wurde ferner bewiesen, daß die gleiche zyklische Aminosäure auch bei der Hydrolyse durch Alkali gebildet wird. ${ }^{1}$ ) Es lag deshalb die Vermutung nahe, daß dieselbe ebenso, wie die gewöhnlichen Aminosäuren, ein primäres Spaltprodukt der Proteinstoffe sei. Ungleich sicherer würde dieser Schluß werden, wenn es gelänge, auch bei der enzymatischen Hydrolyse der Proteinkörper Pyrrolidincarbonsäure zu gewinnen. Ein derartiger Versuch, welcher von Herrn P. Levene im hiesigen Institut ausgeführt wurde, ist von dem einen von uns bereits flüchtig erwähnt worden. ${ }^{2}$ ) Es wurde damals eine sehr kleine Quantität von Pyrrolidincarbonsäure gefunden, aber erst nachdem zu ihrer Isolierung die Überführung in Ester mit Hilfe von Salzsäure angewandt worden war. Da die Möglichkeit nicht ausgeschlossen ist, $\mathrm{da} B$ bei der Veresterung in geringem Maße eine weitergehende Hydrolyse durch die Salzsäure eintritt, so haben wir den Versuch mit größerer Vorsicht wiederholt. Beim Casein, das am ausführlichsten studiert wurde, ergab sich folgendes überraschende Resultat. Die Verdauung durch Pankreasenzyme gibt, auch wenn sie monatelang fortgesetzt wird, keine mit den jetzigen Methoden (d. h. ohne die Veresterung) nachweisbare Menge von

1) Fischer, Emil, Notizen. I. Bildung von $\alpha$-Pyrrolidincarbonsäure bei der Hydrolyse des Caseins durch Alkali., Diese Zeitschr., Bd. XXXV, S. 227, 1902.

2) Fischer, Emil, Über die Hydrolyse des Caseins durch Salzsäure. Diese Zeitschr., Bd. XXXIII, S. 170, 1901.

Hoppe-Seyler's Zeitschrift f. physiol. Chemie. XXXIX. 
Pyrrolidincarbonsäure, dagegen findet sich in der Verdauungsflüssigkeit ein polypeptidartiger Stoff, der offenbar dem Enzym gänzlich widersteht, und der beim Kochen mit Salzsäure fast ebenso große Mengen von $\alpha$-Pyrrolidincarbonsäure liefert, wie die entsprechende Menge Casein selbst. Genau dasselbe gilt merkwürdigerweise für das Phenylalanin.

Als Ferment verwandten wir nicht das gewöhnliche käufliche Trypsin (Grübler), sondern das sog. Pankreatin von der Firma Rhenania in Aachen, das durch größere Wirksamkeit ausgezeichnet, ist. Unter den später angeführten Bedingungen gestaltet sich die Verdauung des Caseins in folgender Weise. Zuerst wird Tyrosin bemerkbar, dessen Krystallisation nach mehreren Stunden beginnt und nach 1-2 Tagen beendet ist. Langsamer erscheinen dann in der Verdauungsflüssigkeit Leucin, Alanin, Asparaginsäure und Glutaminsäure und, wie aus ältern Untersuchungen bekannt ist, auch die drei Diaminosäuren.

Die Biuretreaktion, die anfangs sehr stark ist und im Laufe der Zeit immer schwächer wird, war z. B. nach 6 Wochen anscheinend nicht stärker, als wie es der angewandten Menge Pankreatin entspricht. Auch nach 7 monatlicher Verdauung waren $\alpha$-Pyrrolidincorbonsäure und Phenylalanin in der Flüssigkeit nicht nachweisbar. Sie finden sich im oben erwähnten polypeptidartigen Stoff. Dieser ist so leicht durch Phosphorwolframsäure fällbar, daß er leicht von den Monoaminosäuren getrennt werden kann. Durch mehrmalige Fällung mit Phosphorwolframsäure läßt er sich soweit reinigen, daß er die Biuretreaktion entweder gar nicht oder doch nur äußerst schwach liefert. Das Produkt ist also kein gewöhnliches Pepton. Bei seiner Hydrolyse durch kochende Salzsäure entstehen außer $\alpha$-Pyrrolidincarbonsäure und Phenylalanin auch die übrigen als Spaltprodukte des Caseins bekannten Monoaminosäuren: Leucin, Alanin, Glutaminsäure und Asparaginsäure. Ob auch die Diaminosäuren hierhin zu zählen sind, müssen wir einstweilen unentschieden lassen.

Dieselben Erscheinungen, wie beim Casein, haben wir beim Edestin, Hämoglobin, Eieralbumin, Fibrin und Serumglobulin beobachtet. 
Das beim Casein von uns erhaltene Resultat steht im Widerspruch mit den bisherigen Anschauungen der Physiologen. Aus dem Umstande, daß Tyrosin, Leucin, Glutaminsäure und Asparaginsäure und ferner die Diaminosäuren bei der tryptischen Verdauung nach relativ kurzer Zeit in Freiheit gesetzt wurden, hat man geschlossen, daß ein Totalzerfall des Moleküls bis zu diesen Spaltprodukten eintrete. Unsere Beobachtungen zeigen aber, daß die Hydrolyse unter solchen Bedingungen stets nur partiell ist, und daß schließlich ein widerstandsfähiger Rest bleibt, der zwar nicht mehr Pepton, aber doch noch ein kompliziertes Polypeptid ist und alle die Monoaminosäuren, die überhaupt bisher im Casein gefunden wurden, enthält. Da die fünf andern, oben erwähnten Eiweißkörper sich ähnlich verhalten, so haben wir es hier wahrscheinlich mit einem ganz allgemeinen Phänomen zu tun, dessen biologische Bedeutung sich augenblicklich nach ihrem ganzen Umfang noch nicht übersehen läßt. Jedenfalls wird dadurch die in neuerer Zeit vielfach ausgesprochene Ansicht, daß die Eiweißkörper im Darm völlig bis zu den Aminosäuren hydrolysiert werden, in hohem Grade unwahrscheinlich. Glücklicherweise besitzen wir in dem scharfen Nachweis der Pyrrolidincarbonsäure und des Phenylalanins ein bequemes Mittel, das Vorhandensein jenes interessanten Polypeptids festzustellen. Ob dasselbe ein einheitliches Individuum, oder ein Gemisch ähnlicher Stoffe ist, haben wir vorläufig noch nicht geprüft. Ebenso offen bleibt die Frage, ob das betreffende Produkt für die verschiedenen Eiweißkörper das gleiche ist. Endlich geben unsere Versuche auch nicht die gesuchte definitive Entscheidung bezüglich der Bildung der $\alpha$-Pyrrolidincarbonsäure. Da sie bei der enzymatischen Spaltung der Eiweißkörper nicht in Freiheit gesetzt wird, so bleibt noch immer die Möglichkeit, daß sie sekundär durch die Wirkung von Säure oder Alkali bei höherer Temperatur aus einem bisher noch unbekannten Bestandteil des Eiweißmoleküls entsteht. Für wahrscheinlich halten wir dies allerdings nicht, besonders mit Rücksicht auf das Phenylalanin, bei welchem die Verhältnisse genau ebenso liegen; denn dieses hat seiner chemischen Zusammensetzung nach ebenso das Recht, als 
direkter Bestandteil des Eiweißmoleküls angesehen zu werden, wie die übrigen Monoaminosäuren.

Unsere Beobachtungen zeigen, daß auch bei den gewöhnlichen Eiweißkörpern hydrolytische Spaltprodukte auftreten, die zwischen den Peptonen und den Aminosäuern stehen. Sie schließen sich deshalb auf das engste den Resultaten an, welche bei der Hydrolyse des Seidenfibroins früher erhalten wurden. ${ }^{1}$ ) Hier wurde zum erstenmal die kombinierte Wirkung von Säuren, Fermenten und Basen zur Spaltung eines Proteinstoffes benutzt, und dadurch eine Serie von nicht weniger als vier Zwischenprodukten zwischen den ursprünglichen Proteinstoffen und den Aminosäuren beobachtet. Die Wirkung der Salzsäure gab zunächst das schon bekannte Sericoin, dann bei längerer Dauer der Operation ein peptonartiges Produkt, welches Tyrosin enthält. Durch Trypsin oder Pankreatin konnte aus diesem das Tyrosin abgespalten und ein neuer peptonartiger Stoff erhalten werden, der keine Spur von Tyrosin mehr enthielt. Als letzterer dann vorsichtig mit warmem Barytwasser zerlegt wurde, entstand unter anderen ein Körper, der höchst wahrscheinlich in die Klasse der Dipeptide gehört. Wir zweifeln nicht daran, daß die Übertragung dieser Methoden auf die gewöhnlichen Eiweißkörper zu ähnlichen Resultaten führen wird, und daß es dereinst, wenn bessere Isolierungsmethoden für die Polypeptide gefunden sind, gelingen wird, den hydrolytischen Abbau der Proteine in einer noch viel größern Anzahl von Stufen durchzuführen.

Die Beobachtung, daß ein Teil des Eiweißmoleküls der vollständigen Hydrolyse durch Pankreasenzyme starken Widerstand leistet, ist keineswegs neu.. Wir erinnern an die Ver-

1) Emil Fischer und Peter Bergell, Vortrag auf der Naturforscherversammlung in Karlsbad. Sept. 1902. Ein von mir verfaßtes Referat über diesen Vortrag findet sich unter dem Titel «Über die Hydrolyse der Proteinstoffe» in der Chemiker-Zeitung, 4. Okt. 1902, Jahrg. XXVI, Nr. 80. Die ausführliche Publikation über diese Versuche ist durch längere Krankheit von Dr. Bergell und durch den Wunsch, das dipeptidartige Produkt synthetisch zu gewinnen, verzögert worden. Sie soll aber in nächster Zeit erfolgen.

E. Fischer. 
suche von $\left.K u ̈ h n e,{ }^{1}\right)$ der für seine Antipeptone gerade diese Eigenschaften hervorhebt, ferner an die Mitteilung von Pick, ${ }^{2}$ ) und endlich an die in neuester Zeit erschienenen Arbeiten von M. Siegfried ${ }^{3}$ ) und seinen Schülern. Dieser kommt zu dem Schluß, «daß bei der Einwirkung von Trypsin auf Eiweiß ein Teil desselben unter Bildung von Amidosäuern und Basen leicht zersetzt wird, und daß hierbei Peptone gebildet werden, welche die Tyrosingruppe nicht enthalten und der weiteren Aufspaltung durch Trypsin hartnäckig widerstehen». Dieser Satz ist richtig, wenn die Verdauung nicht allzu lange fortgesetzt wird. Im andern Falle aber werden die Peptone weiter gespalten, wie das Verschwinden der Biuretreaktion zeigt, und es resultiert schließlich das polypeptidartige Produkt, welches von uns als Derivat der $\alpha$-Pyrrolidincarbonsäure und des Phenylalanins gekennzeichnet ist. Besondere Beachtung verdient noch die feste Bindung des Phenylalanins in den Proteinstoffen im Gegensatz zu dem so nah verwandten Tyrosin. Letzteres erscheint in der Regel zuerst als Spaltungsprodukt bei der Verdauung von Eiweißkörpern. Bei dem oben erwähnten Pepton aus Seidenfibroin erfolgt die Abspaltung des Tyrosins durch Trypsin so rasch, daß das Phänomen fast wie eine Krystallisation der Aminosäure aus warmem Wasser aussieht. Ähnliche Erfahrungen, über die an anderer Stelle berichtet werden soll, haben die Versuche mit synthetischen Polypeptiden, welche Tyrosin enthalten, gegeben.

Wir beabsichtigen, die Produkte, die bei der partiellen Spaltung der Proteinstoffe mit Säuren entstehen, in ähnlicher Art auf ihren Gehalt an Pyrrolidincarbonsäure und Phenyl-

1) W. Kühne, Verdauung der Eiweißstoffe durch den Pankreassaft. Virchows Archiv, Bd. 39, 130, 1867. Jd. Weitere Mitteilungen über die Verdauungsenzyme und die Verdauung der Albumine. Verhdl. des Heidelberger naturhist.-mediz. Vereins. N. F. I, 236, 1876. W. Kühne und R. H. Chittenden, Die nächsten Spaltungsprodukte der Eiweißkörper. Zeitschr. f. Biologie, Bd. 19, 159, 1883. Dieselben: Über die Peptone. Zeitschr. f. Biologie, Bd. 22, 423, 1885.

2) Pick, E. P., Zur Kenntnis der peptischen Spaltungsprodukte des Fibrins. Diese Zeitschr., Bd. XXVIII, S. 219, 1899.

3) M. Siegfried, Über Peptone. Diese Zeitschr., Bd. XXXVIII, S. 259, 1903. 
alanin zu prüfen. Das scheint uns um so notwendiger, als nach den Mitteilungen von M. Siegfried ${ }^{1}$ ) die Spaltung durch Säuren in anderem Sinne zu verlaufen scheint, als diejenige mit Enzymen.

Ferner verdient die Frage, ob die kombinierte Wirkung von Pepsin bezw. Papagotin und von Trypsin das gleiche Resultat wie die Trypsinverdauung allein hat, sorgfältig geprüft zu werden.

\section{Experimenteller Teil.}

Bei der Verdauung der Eiweißkörper durch Trypsin oder Pankreatin entsteht $\alpha$-Pyrrolidincarbonsäure nicht in solcher Menge, daß sie mit den jetzigen Methoden erkannt werden kann.

1. $100 \mathrm{~g}$ Casein. pur. (Hammarsten) wurden in $1000 \mathrm{ccm}$ Wasser suspendiert, mit $2 \mathrm{ccm}$ Ammoniak, Toluol und Chloroform versetzt und 8 Tage lang mit $2 \mathrm{~g}$ Pankreatinum purum (Rhenania) im Brutraum bei $36-37^{\circ}$ verdaut. Die vom ausgeschiedenen Tyrosin und Leucin abfiltrierte Flüssigkeit wurde hierauf im Vacuum bei einer $50^{\circ}$ nicht übersteigenden Temperatur auf die Hälfte eingedampft, mit Alkohol gefällt, vom Niederschlag abfiltriert und weiter eingeengt. Die Alkoholfällung wurde 5-6mal wiederholt. Der zuletzt verbleibende alkoholische Auszug wurde eingedampft. Der Rückstand roch nach Indolderivaten. Derselbe wurde mit gefälltem Kupferoxyd gekocht. Das sich bildende Kupfersalz ließ sich in einen alkohollöslichen und einen alkoholunlöslichen Teil zerlegen, aber Pyrrolidincarbonsäure war in keinem der beiden Teile mit Sicherheit nachweisbar.

Ganz dasselbe Resultat gaben die folgenden Versuche 2-6.

2. $100 \mathrm{~g}$ Casein. pur. (H.) wurden in $1500 \mathrm{ccm}$ Wasser suspendiert, mit $2 \mathrm{ccm}$ Ammoniak, Toluol und Chloroform versetzt und 8 Tage lang mit $4 \mathrm{~g}$ Pankreatin verdaut.

3. $200 \mathrm{~g}$ Casein in derselben Weise mit $2 \times 3 \mathrm{~g}$ Pankreatin 14 Tage verdaut. Flüssigkeitsmenge $2000 \mathrm{ccm}$.

4. $250 \mathrm{~g}$ Casein 14 Tage mit $3 \times 2^{1 / 2} \mathrm{~g}$ Pankreatin verdaut. Flüssigkeitsmenge $2000 \mathrm{ccm}$.

1) M. Siegfried, Zur Kenntnis der Hydrolyse des Eiweißes. Berichte der math.-phys. Klasse der kgl. sächs. Gesellsch. f. Wissenschaften zu Leipzig. Sitzung vom 2. III. 1903. 
5. $500 \mathrm{~g}$ Casein in $5000 \mathrm{ccm}$.Wasser suspendiert und mit $10 \mathrm{~g}$ Pankreatin während 50 Tagen verdaut. $100 \mathrm{~g}$ des Verdauungsgemisches wurden nach dieser Zeit in der oben geschilderten Weise untersucht.

6. 200 g Plasmon (Caseinnatron) waren 7 Monate lang mit $4 \mathrm{~g}$ Pankreatin verdaut, und hierauf noch weitere 14 Tage mit nochmals $4 \mathrm{~g}$ Pankreatin im Brutraum stehen gelassen worden. Flüssigkeitsmenge $2000 \mathrm{ccm}$. Die Biuretreaktion war hier bei dem Niederschlag mit Phosphorwolframsäure ganz verschwunden.

Unter denselben Bedingungen wurden Edestin, Serum globulin, Eieralbumin, Hämoglobin und Fibrin untersucht.

a) Edestin $(100 \mathrm{~g}) 12$ Wochen mit $2 \times 2,5 \mathrm{~g}$ Pankreatin verdaut. Flüssigkeitsmenge $1000 \mathrm{ccm}$.

b) Serumglobulin $(100 \mathrm{~g}) 25$ Tage mit $5 \times 2$ g Pankreatin. Flüssigkeitsmenge $1000 \mathrm{ccm}$.

c) Eieralbumin $(100 \mathrm{~g}) 21$ Tage mit $2 \times 2$ g Pankreatin. Flüssigkeitsmenge $1000 \mathrm{ccm}$.

d) Hämoglobin $(100 \mathrm{~g}) 12$ Wochen mit $3 \times 2 \mathrm{~g}$ Pankreatin. Flüssigkeitsmenge $1000 \mathrm{ccm}$.

e) Fibrin (100 g) wurden mit $2 \mathrm{~g}$ Pankreatin eine Woche lang verdaut. Flüssigkeitsmenge $1000 \mathrm{ccm}$.

In allen Fällen war es unmöglich, freie Pyrrolidincarbonsäure nach dem geschilderten Verfahren nachzuweisen.

Bei der Verdauung des Caseins entsteht neben Tyrosin, Leucin, Alanin, Asparaginsäure und Glutaminsäure als Endprodukt ein polypeptidartiger Stoff, der bei weiterer Hydrolyse mit kochender Salzsäure reichliche Mengen von $\alpha$-Pyrrolidincarbonsäure und Phenylalanin liefert.

1. $100 \mathrm{~g}$ Casein. pur. (Hammarsten) wurden in $1000 \mathrm{ccm}$ Wasser suspendiert, mit $2 \mathrm{ccm}$ Ammoniak, Toluol und Chloroform versetzt und 8 Tage lang im Brutraum bei $36-37^{\circ}$ mit $3 \mathrm{~g}$ Pankreatin verdaut. Die Biuretreaktion war deutlich vorhanden. ${ }^{1}$ ) Nachdem das ausgeschiedene Tyrosin abfiltriert

1) Bei der Ausführung der Biuretreaktion ist darauf zu achten, daß genügend Alkali vorhanden ist. Ferner scheint es nicht überflüssig, darauf hinzuweisen, daß Pankreatin selbst eine intensive Biuretreaktion gibt. 
war, wurde die Flüssigkeit zur Vertreibung des freien Ammoniaks bei einer $50^{\circ}$ nicht übersteigenden Temperatur im Vacuum eingeengt, dann auf $2000 \mathrm{ccm}$ gebracht und mit Phosphorwolframsäure im Überschuß gefällt. ${ }^{1}$ ) Der Niederschlag wurde abgesaugt, dann mit der hydraulischen Presse ausgepreßt, nun in einer Reibschale fein gepulvert, in Wasser suspendiert und mit überschüssigem Baryt während 24 Stunden bei gewöhnlicher Temperatur geschüttelt. Nachdem der phosphorwolframsaure Baryt abgesaugt und hydraulisch abgepreßt war, wurde das Filtrat mit Schwefelsäure vom überschüssigen Baryt befreit, dann nochmals mit überschüssiger Phosphorwolframsäure gefällt, und der Niederschlag in genau derselben Weise, wie oben geschildert, abgepreßt und zerlegt.

Die vereinigten Filtrate von den beiden Phosphorwolframsäurefällungen wurden im Vacuum eingedampft und der Rückstand hierauf mit rauchender Salzsäure 6 Stunden am Rückflußkühler gekocht, um alle etwa noch vorhandenen Polypeptide zu spalten. Die Flüssigkeit färbte sich hierbei violett. Sie wurde nun im Vacuum zum Sirup eingedampft, und letzterer zum Nachweis der Aminosäuern in der bekannten Weise verestert.

Die Destillation der Ester gab folgende Fraktionen:

1. 0 bis $40^{\circ}$ (Temperatur des Wasserbades) bei $12 \mathrm{~mm}$ Druck: $1,0 \mathrm{~g}$ 2. $40 \times 100^{\circ}(">)>12,>4,0$, $3 .>100^{\circ}(*>)>0,2>>10,5$, 4. $100 \times 160^{\circ}$ ( $>$ Ölbades $) \times 0,2$ > 12,5 *

Isoliert wurden $0,2 \mathrm{~g}$ Alanin, $5 \mathrm{~g}$ Leucin, $3 \mathrm{~g}$ Glutaminsäure, 0,5 g Asparaginsäure.

Phenylalanin und Pyrrolidincarbonsäure wurden nicht aufgefunden. Auf die Isolierung des Tryptophans, dessen Reaktion

1) Diese starke Verdünnng und der. Überschuß der Phosphorwolframsäure wurden in Hinsicht auf die Angaben von E. Schulze und E. Winterstein (Trennung des Phenylalanins von anderen Animosäuren. Diese Zeitschr., Bd XXXV, S. 123, 1902) verwendet. Casein enthält ca. 3 bis $31 / 2 \%$ Phenylalanin. Nach E. Schulze und E. Winterstein wird eine ca. $0,5 \%$ oige Lösung von Phenylalanin bei Gegenwart von Leucin mit Phosphorwolframsäure nicht mehr gefällt. Eigene Versuche bestätigten diese Angaben. 
die Flüssigkeiten stark zeigten, sowie der Oxyaminosäuren haben wir verzichtet.

Der zerlegte Phosphorwolframsäureniederschlag wurde nach dem Absaugen und Abpressen des phosphorwolframsauren Baryts mit Schwefelsäure vom überschüssigen Baryt befreit, hierauf im Vacuum eingedampft. Der Rückstand war anfangs ein Sirup, der aber zu einer amorphen, schwach gelb gefärbten leimartigen Masse eintrocknete. Er löste sich äußerst leicht in Wasser und fiel auf Zusatz von Alkohol in amorphen Flocken wieder aus. Wir werden später versuchen, dieses Rohprodukt, welches aller Wahrscheinlichkeit nach Arginin, Lysin und Histidin enthält, weiter zu reinigen. Für den vorliegenden Zweck war dies überflüssig, da die Diaminosäuren, wie wir nochmals durch einen besonderen Versuch festgestellt haben, beim Kochen mit Salzsäure keine Pyrrolidincarbonsäure liefern. Dieser amorphe polypeptidartige Rückstand wurde nun zur Hydrolyse mit rauchender Salzsäure am Rückflußkühler während 6 Stunden gekocht, wobei sich die Lösung ebenso stark dunkelviolett färbte, wie bei Anwendung des Caseins selbst. Die Flüssigkeit wurde schließlich im Vacuum zum Sirup eingeengt und verestert.

Bei der Destillation des Estergemisches resultierten folgende Fraktionen :

1. 0 bis $40^{\circ}$ (Temperatur des Wasserbades) bei $12 \mathrm{~mm}$ Druck: $0,5 \mathrm{~g}$

2. $40 \cdot 100^{\circ}(>, \quad), 12,>1,5$, 3. $>100^{\circ}(>, \quad) 0,3 \times>4,0$, 4. $100 \rightarrow 160^{\circ}(>\quad$ Ölbades $)>0,3>17,0$,

Isoliert wurden $0,1 \mathrm{~g}$ Alanin, $2 \mathrm{~g}$ Leucin, $4 \mathrm{~g}$ Glutaminsäure, $1 \mathrm{~g}$ Asparaginsäure, ferner 1,5 g $\alpha$-Pyrrolidincarbonsäure und 2,2 g Phenylalanin. ${ }^{1}$ )

Analyse der Pyrrolidincarbonsäure:

0,1002 g Substanz gaben 0,1912 $\mathrm{g} \mathrm{CO}_{2}$ und $0,0713 \mathrm{~g} \mathrm{H}_{2} \mathrm{O}$.

Berechnet für $\mathrm{C}_{5} \mathrm{H}_{9} \mathrm{NO}_{2}$ :

Gefunden:

$52,18 \% \mathrm{C}$ und $7,83 \% \mathrm{H}$. $52,04 \% \mathrm{C}$ und $7,97 \% \mathrm{H}$.

Schmelzpunkt gegen $205^{\circ}$ (unkorr.)

1) Die Methode der Isolierung betreffend vergl.: Emil Fischer und Emil Abderhalden, Hydrolyse des Oxyhämoglobins durch Salzsäure. Diese Zeitschr., Bd. XXXVI, Heft 4, S. 273, 1902. 
Das Phenylalanin gab folgende Zahlen:

$0,2061 \mathrm{~g}$ Substanz gaben $0,4953 \mathrm{~g} \mathrm{CO}_{2}$ und $0,1251 \mathrm{~g} \mathrm{H}_{2} \mathrm{O}$.

Berechnet für $\mathrm{C}_{8} \mathrm{H}_{11} \mathrm{NO}_{8}$ :

$65,45 \% \mathrm{C}$ und $6,66 \% \mathrm{H}$.

Gefunden:

Schmelzpunkt gegen $283^{\circ}$ (unkorr.)

2. $100 \mathrm{~g}$ Casein. pur. (H.) wurden in $1000 \mathrm{ccm}$ Wasser suspendiert, unter ganz den gleichen Bedingungen wie in Versuch 1 mit $3 \mathrm{~g}$ Pankreatin 8 Tage lang verdaut und die Verdauungsflüssigkeit genau so wie bei Versuch 1 verarbeitet.

Die vereinigten Filtrate von den beiden Phosphorwolframsäureniederschlägen gaben nach erfolgter Veresterung des Rückstandes folgendes Resultat:

Fraktion 1: bis $100^{\circ}$ (Temp. d. Wasserbades) bei $12 \mathrm{~mm}$ Druck: $3,5 \mathrm{~g}$

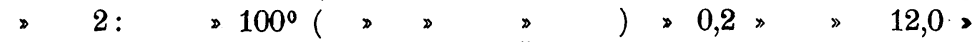

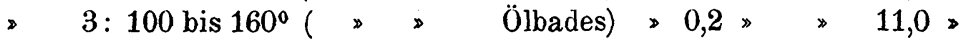

Isoliert wurden 0,1 g Alanin, 5,5 g Leucin, $3 \mathrm{~g}$ Glutaminsäure, $1 \mathrm{~g}$ Asparaginsäure.

Phenylalanin und Pyrrolidincarbonsäure wurden nicht erhalten.

Die zweite Phosphorwolframsäurefällung zeigte nur ganz schwache Biuretreaktion und gab folgende Esterfraktionen:

Fraktion 1: bis $100^{\circ}$ (Temp. d. Wasserbades) bei $12 \mathrm{~mm}$ Druck : $2,5 \mathrm{~g}$

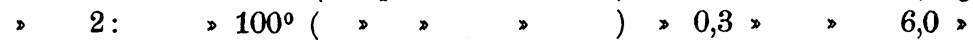

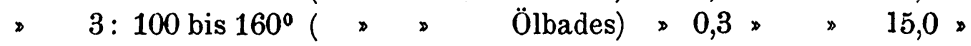

Isoliert wurden $0,3 \mathrm{~g}$ Alanin, $3 \mathrm{~g}$ Leucin, 2,5 g Glutaminsäure, $0,8 \mathrm{~g}$ Asparaginsäure und ferner $2,2 \mathrm{~g}$ Pyrrolidincarbonsäure und $2,8 \mathrm{~g}$ Phenylalanin.

Das isolierte Phenylalanin schmolz gegen $280^{\circ}$ (unkorr.) und hatte die richtige Zusammensetzung.

$0,2101 \mathrm{~g}$ Substanz gaben $0,5050 \mathrm{~g} \mathrm{CO}_{2}$ und 0,1255 $\mathrm{g} \mathrm{H}_{2} \mathrm{O}$.

Berechnet für $\mathrm{C}_{9} \mathrm{H}_{11} \mathrm{NO}_{2}$ :

Gefunden:

$65,45 \% \mathrm{C}$ und $6,66 \% \mathrm{H}$.

$65,55 \% \mathrm{C}$ und $6,69 \% \mathrm{H}$.

Die Analyse der gegen $207^{\circ}$ (unkorr.) schmelzenden $\alpha$-Pyrrolidincarbonsäure gab folgende Zahlen:

$0,2012 \mathrm{~g}$ Substanz gaben $0,3844 \mathrm{~g} \mathrm{CO}_{\mathbf{2}}$ und $0,1432 \mathrm{~g} \mathrm{H}_{\mathbf{z}} \mathrm{O}$.

Berechnet für $\mathrm{C}_{5} \mathrm{H}_{9} \mathrm{NO}_{8}$ :

Gefunden :

$52,18 \% \mathrm{C}$ und $7,83 \% \mathrm{H} . \quad 52,10 \% \mathrm{G}$ und $7,97 \% \mathrm{H}$.

3. 200 g Casein. pur. (H.) wurden mit $2 \times 2$ g Pankreatin 14 Tage lang verdaut. Die Verdauungsflüssigkeit gab eine ganz schwache Biuretreaktion. 
Auch bei diesem Versuche konnte im Filtrate der Phosphorwolframsäurefällung weder Phenylalanin noch Pyrrolidincarbonsäure nachgewiesen werden.

Aus der zweiten Phosphorwolframsäurefällung wurden 5,2 g Phenylalanin (Schmelzpunkt $281^{\circ}$ [unkorr.]) und 6,2 g $\alpha$-Pyrrolidincarbonsäure (Schmelzpunkt $204^{0}$ [unkorr.]) erhalten.

4. $250 \mathrm{~g}$ Casein. pur. (H.) mit $4 \times 2$ g Pankreatin während 14 Tagen verdaut.

Ausgeschieden hatten sich $14 \mathrm{~g}$ Leucin und Tyrosin. Die Biuretreaktion war vorhanden, aber sehr schwach.

Das Filtrat vom Phosphorwolframsäureniederschlag ergab nach der Veresterung folgende Fraktionen:

Fraktion 1: 0 bis $100^{\circ}$ (Temp. d. Wasserbades) bei $14 \mathrm{~mm}$ Druck: $5,0 \mathrm{~g}$

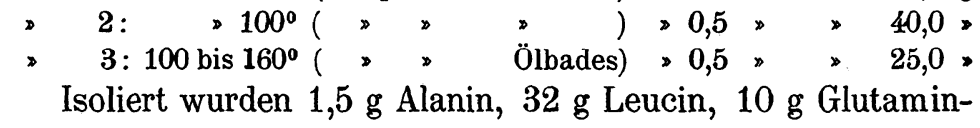
säure und $3 \mathrm{~g}$ Asparaginsäure. Auch in diesem Versuche wurde keine Spur Phenylalanin und Pyrrolidincarbonsäure gefunden.

Der zerlegte Phosphorwolframsäureniederschlag gab folgende Fraktionen:

Fraktion 1: 0 bis $100^{\circ}$ (Temp. d. Wasserbades) bei $13 \mathrm{~mm}$ Druck: $3,0 \mathrm{~g}$

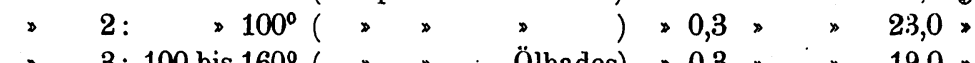
> 3: 100 bis $160^{\circ}(\triangleright>$ Ölbades $)>0,3 \gg, 19,0$,

Isoliert wurden $1 \mathrm{~g}$ Alanin, $10 \mathrm{~g}$ Leucin, $6 \mathrm{~g}$ Glutaminsäure, 1,5 g Asparaginsäure, 5,2 g Pyrrolidincarbonsäure und $6 \mathrm{~g}$ Phenylalanin.

5. $150 \mathrm{~g}$ Casein. pur. (H.) wurden 4 Wochen mit $2 \times 3 \mathrm{~g}$ Pankreatin verdaut. Die Biuretreaktion war nur schwach angedeutet. Während das Filtrat vom Phosphorwolframsäureniederschlag keine Spur von Phenylalanin und Pyrrolidincarbonsäure gab, wurden aus dem Niederschlag selbst $4 \mathrm{~g}$ Pyrrolidincarbonsäure (Schmelzpunkt $206^{\circ}$ [unkorr.]) und $5 \mathrm{~g}$ Phenylalanin (Schmelzpunkt $281^{\circ}$ [unkorr.]) isoliert.

6. $500 \mathrm{~g}$ Casein. pur. (H.) wurden mit $10 \mathrm{~g}$ Pankreatin während 50 Tagen verdaut. Die Biuretreaktion war noch vorhanden, aber sehr schwach. Von dieser Verdauungsflüssigkeit wurden $1 / 5$ zur direkten Prüfung auf Pyrrolidincarbonsäure und 2/5 zur Phosphorwolframsäurefällung verwendet; die übrigen 
2/5 wurden mit $4 \mathrm{~g}$ Pankreatin noch 2 Wochen lang verdaut, und hierauf ebenfalls mit Phosphorwolframsäure gefällt.

In der nach 50 Tagen entstandenen Verdauungsflüssigkeit war das Resultat genau den in den vorigen Versuchen erhaltenen entsprechend.

Die nach 64 Tagen untersuchte Probe gab nur noch eine äußerst schwache Biuretreaktion. Das Filtrat vom Phosphorwolframsäureniederschlag enthielt kein Phenylalanin, dagegen konnten 0,23 g Pyrrolidincarbonsäure, die wohl aus dem in Lösung gebliebenen Polypeptid durch das Kochen mit Salzsäure entstanden war, isoliert werden.

Der zerlegte Phosphorwolframsäureniederschlag enthielt 5,2 g Phenylalanin und 5,8 g Pyrrolidincarbonsäure.

7. $100 \mathrm{~g}$ Casein. pur. (H.) wurden mit $4 \times 2$ g Pankreatin während 8 Wochen verdaut. Die Biuretreaktion war nur noch angedeutet. Die Phosphorwolframsäurefällung gab genau dasselbe Resultat, wie die bereits mitgeteilten Versuche.

8. $200 \mathrm{~g}$ Plasma (käufliches Casein-Natron) waren 7 Monate lang mit $4 \mathrm{~g}$ Pankreatin verdaut worden. Die Biuretreaktion war nur noch sehr schwach vorhanden. Nach neuem Zusatz von $4 \mathrm{~g}$ Pankreatin und erneuter 3 Wochen dauernder Verdauung gab der Niederschlag mit Phosphorwolframsäure keineBiuretreaktion mehr.

Bei der Veresterung des Rückstandes, den das Filtrat vom Phosphorwolframsäureniederschlag hinterließ, wurden weder Phenylalanin noch Pyrrolidincarbonsäure gefunden.

Der Phosphorwolframsäureniederschlag selbst enthielt: Alanin, Leucin, Glutaminsäure, Asparaginsäure, Phenylalanin $(2,2 \mathrm{~g})$ und Pyrrolidincarbonsäure $(1 \mathrm{~g})$.

Ähnliche Resultate wie beim Casein erhielten wir beim Edestin, Hämoglobin, Serumglobulin, Eieralbumin und Fibrin.

Die Verdauung wurde unter genau den gleichen Bedingungen angestellt.

a) $100 \mathrm{~g}$ Edestin mit $2 \times 2,5 \mathrm{~g}$ Pankreatin 12 Wochen lang verdaut.

b) $100 \mathrm{~g}$ Serumglobulin 25 Tage mit $5 \times 2$ g Pankreatin.

c) $100 \mathrm{~g}$ Eieralbumin 21 Tage lang mit $2 \times 2 \mathrm{~g}$ Pankreatin. 
Über die Verdauung einiger Eiweißkörper durch Pankreasfermente. 93 Pankreatin.

d) $100 \mathrm{~g}$ Hämoglobin 12 Wochen lang mit $3 \times 2 \mathrm{~g}$

e) 100 g Fibrin mit 2 g Pankreatin während einer Woche.

Die Biuretreaktion war in allen Fällen noch vorhanden, aber meistens schwach und würde wahrscheinlich bei längerer Dauer der Versuche verschwunden sein.

Hervorzuheben ist, daß im Gegensatz zum Fibrin, Edestin, Eieralbumin und Hämoglobin das Serumglobulin dem Pankreatin einen erheblich größeren Widerstand leistete und eine viel größere Menge von Produkten, die durch Phosphorwolframsäure fällbar waren, lieferte.

In allen Fällen fanden sich Phenylalanin und Pyrrolidincarbonsäure nur im Phosphorwolframsäureniederschlag, ebenso war das Glycocoll, welches bekanntlich aus Edestin und Serumglobulin entsteht, ausschließlich im Phosphorwolframsäureniederschlag vorhanden.

Bei obigen Versuchen wurde die Trennung der Monoaminosäuren von dem nur partiell verdauten Teil des Proteinmoleküls durch Phosphorwolframsäure bewerkstelligt. Da in der Literatur verschiedene Angaben existieren, daß diese Methode ungenaue Resultate gebe, und da ferner E. Schulze und E. Winterstein (l. c.) darauf aufmerksam gemacht haben, daß das Phenylalanin bei stärkerer Konzentration durch Phosphorwolframsäure gefällt wird, so haben wir einen besonderen Versuch angestellt, um die Brauchbarkeit der Methode zu prüfen.

$100 \mathrm{~g}$ Casein wurden mit der dreifachen Menge Salzsäure 6 Stunden lang am Rückflußkühler gekocht, und hierauf, nachdem die ganze Flüssigkeit auf $1500 \mathrm{ccm}$ gebracht worden war, mit Phosphorwolframsäure gefällt. Der Niederschlag wurde hierauf abgesaugt und mit der hydraulischen Presse ausgepreßt. Die letzte Operation ist sehr wichtig, denn aus dem auf der Nutsche abgesaugten und fest gepreßten Niederschlage ließ sich mit der hydraulischen Presse noch fast $1 / 6$ der Gesamtflüssigkeit gewinnen. Der Phosphorwolframsäureniederschlag wurde in der oben geschilderten Weise zerlegt. Nach erfolgter Entfernung des überschüssigen Baryts mit Schwefelsäure wurde die Phosphorwolframsäurefällung wiederholt, und der Niederschlag, wie 
94 Emil Fischer u. Emil Abderhalden, Über die Verdauung etc.

oben geschildert, behandelt. Die schließlich resultierende Flüssigkeit, welche die durch Phosphorwolframsäure gefällten Körper enthielt, wurde im Vacuum eingedampft und der Rückstand verestert. Es konnten aber keine in Äther löslichen Ester isoliert werden, dagegen war beim Versuch, die Ester in Freiheit zu setzen, ein deutlicher Estergeruch wahrnehmbar. Mithin sind sicher nur Spuren von Monoaminosäuren vorhanden gewesen. 